in reducing suicide, especially in those age groups whose members are less likely to attend the accident and emergency department at times of crisis, such as children and the elderly.

Duckworth \& McBride (1996) have reported that $80 \%$ of elderly suicide victims received no psychiatric referrals, and according to Harwood et al (2001), only $15 \%$ of elderly people who died by suicide were under psychiatric care at the time of death.

In our study, analysing coroners' inquests of 200 cases of suicide in old age in Cheshire, 1989-2001 (Salib \& El-Nimr, 2003), the role of primary care was emphasised. Interestingly, even those victims who were known to psychiatric services still preferred to contact their general practitioners (GPs) in the last few weeks before the fatal act.

One conclusion might be that people whose GPs acknowledged their mental health problems and cared to refer them to a specialist service were able to build a more meaningful therapeutic relationship with their doctors and readily contacted them as a final desperate act in the last period of their lives. A well-trained GP can act not only as an effective first point of contact but also a final one!

Duckworth, G. \& McBride, H. (1996) Suicide in old age: a tragedy of neglect. Canadian Journal of Psychiatry 4I, 217-222.

Gairin, I., House, A. \& Owens, D. (2003) Attendance at the accident and emergency department in the year before suicide: retrospective study. British Journal of Psychiatry, 183, 28-33.

Harwood D., Hawton, K., Hope, T., et al (200I) Psychiatric disorder and personality associated with suicide in older people: a descriptive and case-control study. International Journal of Geriatric Psychiatry, 16 155-156.

Salib, E. \& El-Nimr, G. (2003) Gender and utilisation of psychiatric services in elderly suicide. International Journal of Psychiatry in Clinical Practice, in press.

G. El-Nimr Neurobehavioural Unit, Haywood Hospital, High Lane, Burslem, Stoke-on-Trent, Staffordshire ST6 7AG, UK

\section{Assertive outreach in Tyneside}

Priebe et al (2003) have questioned whether the findings of the Pan-London Assertive Outreach Study can be generalised to assertive outreach services in the rest of the UK. Cornwall \& Haveman (2003) evaluated the Newcastle and North Tyneside assertive outreach service using the same research instruments as those in the Pan-London study, so direct comparisons can be made.

After 17 months of operation, the Newcastle and North Tyneside team was similar in size to the London mean $(n=56)$ with a score on the Dartmouth Assertive Community Treatment Scale (Teague et al, 1998) of 3.5 (medium fidelity to the model). The team has care programme approach (CPA) responsibility and small case-loads, operates out of office hours but without dedicated in-patient beds and, at the time of evaluation, no consultant psychiatrist. It thus corresponds to a Cluster B team in the Pan-London study (Wright et al, 2003).

Patient contact frequency and duration was higher than the London mean with an average face-to-face contact of 94 minutes per week. There was also a greater focus on patient engagement, with this being the primary focus in $33.1 \%$ of contacts. Engagement with the service in assertive outreach patients was compared with a random sample of community mental health team (CMHT) patients on enhanced CPA. There was no difference in the level of engagement, raising the possibility that the focus on engagement was having an impact in a previously hard-to-engage patient group.

Similar to the London study (Billings et al, 2003), team members were fairly satisfied with their jobs and most were not experiencing high levels of burnout. Compared with two local CMHTs, assertive outreach staff reported a higher level of personal accomplishment, replicating the Pan-London study finding. Another common finding was that the assertive outreach staff rated lack of support from senior staff in the service as a greater source of stress than did CMHT staff. Team members also identified dual diagnosis as an unmet training need.

Newcastle and North Tyneside patients were more likely than London patients to be White $(86 \% v .45 \%)$ or living alone $(68 \% v$. 52\%). More surprisingly, they had significantly higher levels of alcohol misuse or dependency $(31 \% v .16 \%)$ and drug misuse or dependency $(40 \% \quad v$. $20 \%$ ). This reflects the fact that the Newcastle and North Tyneside service may be managing a more severely ill patient group, with $93 \%$ having experience of compulsory admission and $70 \%$ having had an in-patient admission lasting more than 6 months. Using the mean MARC severity score (Huxley et al, 2000), assertive outreach patients in Newcastle and
North Tyneside had significantly more severe problems than the sample of local CMHT patients on enhanced CPA (7.4 v. $3.4 ; t=6.35, \quad$ d.f. $=83, \quad P<0.01$; mean difference $=4.0,95 \%$ CI 2.7-5.3).

Wright et al (2003) have suggested that the London teams are assertive community treatment-like teams, but that the US assertive community treatment model may not easily translate to the UK context. The Newcastle and North Tyneside data contrast with both the London data and data from the UK700 study (Burns et al, 1999) in terms of the strong focus on patient engagement. Longitudinal studies are needed to determine whether this will actually enhance engagement and whether that improves outcome.

Billings, J., Johnson, S., Bebbington, P., et al (2003) Assertive outreach teams in London: staff experiences and perceptions. Pan-London Assertive Outreach Study, Part 2. British Journal of Psychiatry, 183, 139-147.

Burns, T., Creed, F., Fahy, T., et al (1999) Intensive versus standard case management for severe psychotic illness: a randomised trial. UK 700 Group. Lancet, 353 2185-2189.

Cornwall, P. L. \& Haveman, J. (2003) An Evaluation of the Newcastle and North Tyneside Assertive Outreach Service. Newcastle upon Tyne: University of Newcastle School of Neurology, Neurobiology and Psychiatry.

Huxley, P., Reilly, S., Gater, R., et al (2000) Matching resources to care: the acceptability, validity and interrater reliability of a new instrument to assess severe mental illness (MARC-I). Social Psychiatry and Psychiatric Epidemiology, 35, 312-317.

Priebe, S., Fakhoury, W., Watts, J., et al (2003) Assertive outreach teams in London: patient characteristics and outcomes. Pan-London Assertive Outreach Study, Part 3. British Journal of Psychiatry, 183, 148-154.

Teague, G. B., Bond, G. R. \& Drake, R. E. (1998) Program fidelity in assertive community treatment: development and use of a measure. American journal of Orthopsychiatry, 68, 216-232.

Wright, C., Burns, T., James, P., et al (2003) Assertive outreach teams in London: models of operation. PanLondon Assertive Outreach Study, Part I. British Journal of Psychiatry, 183, 132-138.

P. L. Cornwall Tees \& North East Yorkshire NHS Trust, Fern Lodge CMHC, I53 High Street, Eston, MiddlesbroughTS6 9JQ, UK

\section{Treating maternal depression?}

Cooper et al (2003) reported a randomised trial with mothers with post-partum depression that compared routine primary care, non-directive counselling, cognitivebehavioural therapy (CBT) and psychodynamic therapy and found that psychological therapy improved maternal mood in the short term but the long-term effect was no better than spontaneous remission. 